\title{
Use of the plasma RMS signal for on-line welding quality monitoring
}

\author{
J. Mirapeix, A. Cobo, P.B. García-Allende, O.M. Conde, J.M. Lopez-Higuera \\ Photonics Engineering Group, Univ. de Cantabria, Avda. Los Castros s/n, 39005 Santander, Spain
}

\begin{abstract}
In this paper a new spectroscopic monitoring parameter is proposed for the on-line monitoring of welding processes, the plasma RMS signal, which is determined by considering the contribution from the spectral samples over a particular spectral window. This parameter is directly related to the heat input that can be estimated by measuring both welding voltage and current, but it exhibits a higher sensitivity to the appearance of weld defects. A comparison between the results obtained from the different spectroscopic parameters will be presented, with data from both experimental and field arc-welding tests.
\end{abstract}

Keywords: arc-welding, on-line monitoring, plasma optical spectroscopy, RMS signal

\section{INTRODUCTION}

Although an intense research effort has been performed during the last years in the field of arc and laser welding on-line monitoring, it is nowadays an active area of research, and companies from different sectors are demanding realiable and efficient solutions in this regard. Some examples of these fields of application are the fabrication of heavy components for nuclear power stations, engines for aeronautics or different parts of an automobile.

Several options have been proposed, from machine vision to infrared thermography, analysis of the acoustic emission of the process or sensing of the welding voltage and current. Among these proposals plasma optical spectroscopy has emerged as a promising solution, as the analysis of the different species participating in the plasma allows to obtain a direct indication of the seam quality. The traditional scheme in this approach is the estimation of the plasma electronic temperature $T_{e}$ by means of two or more emission lines from the same species. However, the identification of the emission lines may give rise to some uncertainties, depending on the spectral resolution of the chosen instrumentation.

Some alternatives have been recently proposed, like the use of correlation analysis of the plasma spectra [1], or the generation of participation profiles of the plasma species with synthetic spectra and optimization algorithms [2]. Other solutions are more related to the conventional approach mentioned above, for example the selection of the wavelength associated with the maximum intensity of the plasma continuum [3], or the employment of the line-to-continuum method as an alternative to obtain the plasma temperature [4].

In this paper the use of the plasma spectrum root mean square RMS signal is proposed as an alternative on-line monitoring parameter. A similar approach has been initially explored by Wang et al. [5] for laser welding of titanium alloys by using a photodiode. In our system a CCD spectrometer is employed, and the plasma spectrum RMS signal is calculated by considering the intensity associated with all the pixels in the sensor. With this approach it is possible to provide in real-time different spectroscopic monitoring parameters and, depending on the particular process, to use only one or to combine some of them under specific logic rules. In addition, the use of the CCD spectrometer allows to perform a selection of the spectral window to be analyzed, instead of using the whole spectral range of the chosen instrumentation. Both experimental and field arc-welding tests performed in the facilities of ITP (Industria de Turbo Propulsores S.A.), a company devoted to the fabrication of components for aeronautics, with both Inconel 718 and Titanium $6 \mathrm{Al}-4 \mathrm{~V}$ specimens, will show the feasibility of the proposed solution.

Optical Sensing and Detection, edited by Francis Berghmans, Anna Grazia Mignani, Chris A. van Hoof, Proc. of SPIE Vol. 7726, 772625 - () 2010 SPIE · CCC code: 0277-786X/10/\$18 · doi: 10.1117/12.854684 


\section{PLASMA DIAGNOSTICS APPLIED TO ON-LINE WELDING MONITORING}

As commented in the previous section, different spectroscopic monitoring parameters have been considered for on-line welding quality diagnostics. The traditional variable use for monitoring is the plasma electronic temperature $T_{e}$, which can be determined by means of the Boltzmann-plot, derived from the Boltzmann equation [6]

$$
\ln \left(\frac{I_{m n} \lambda_{m n}}{A_{m n} g_{m}}\right)=\ln \left(\frac{h c N}{Z}\right)-\frac{E_{m}}{k T_{e}}
$$

where $E_{m}$ is the upper level energy, $g_{m}$ the statistical weight, $A$ the transition probability, $\lambda$ the wavelength, $I$ the emission line relative intensity, $k$ the Boltzmann constant, $h$ the Planck's constant, $c$ the light velocity, $N$ the population density of the state $m$ and $Z$ the partition function. The representation of the left-hand side of Equation (1) versus $E_{m}$ has a slope inversely proportional to $T_{e}$. Several emission lines from the same species are considered in this case to obtain the $T_{e}$ profile, but this can be simplified by choosing only two lines and using Equation (2)

$$
T_{e}=\frac{E_{m}(2)-E_{m}(1)}{k \ln \left[\frac{I(1) A(2) g_{m}(2) \lambda(1)}{I(2) A(1) g_{m}(1) \lambda(2)}\right]} .
$$

Equation (2) is commonly employed for on-line welding monitoring, given its reduced computational cost. However, it is worth mentioning that the temperature profiles will be noisier with this approach, what can be a problem for this kind of application.

The RMS signal of the plasma spectrum is calculated by considering the intensity provided by each pixel of the CCD sensor included in the setup

$$
S_{R M S}=\sqrt{\frac{1}{n} \sum_{i=0}^{n-1} x_{i}^{2}}
$$

where $n$ is the total number of pixels of the CCD and $x_{i}$ the intensity corresponding to the $i$-th pixel. This signal is a measurement of the plasma spectrum energy within the spectral range considered, and it is also related to the total arc power, and consequently to the process heat input. This parameter gives a relative measurement of the energy transferred from the welding electrode to the base metal by the electric arc, and can be defined as

$$
H=\frac{V I}{S}
$$

In Equation (4) $V$ is the welding voltage, $I$ the current and $S$ the welding speed. The heat input is an important parameter as it influences the cooling rate, which may affect the mechanical properties and metallurgical structure of the weld and the heat affected zone (HAZ).

It is also worth noting that the determination of $T_{e}$ by means of Equation (2) requires several processing stages, that have been illustrated in Figure 1. All of them require a specific processing time, but the identification of the plasma emission lines is the most demanding in this regard. This process is based on the association of a particular species (element in a particular ionization stage) to an emission line chosen during the process. A common approach is based on the use of a local NIST [7] database with the required spectroscopic information, but this implies a certain uncertainty, especially for industrial applications where low cost spectrometers use to be selected. The employment of the RMS signal deals with these two drawbacks. On the one hand, the solution is more efficient computationally, given that the resulting processing scheme is simplified. On the other hand, the emission lines do not need to be identified, what avoids the uncertainty mentioned above. 


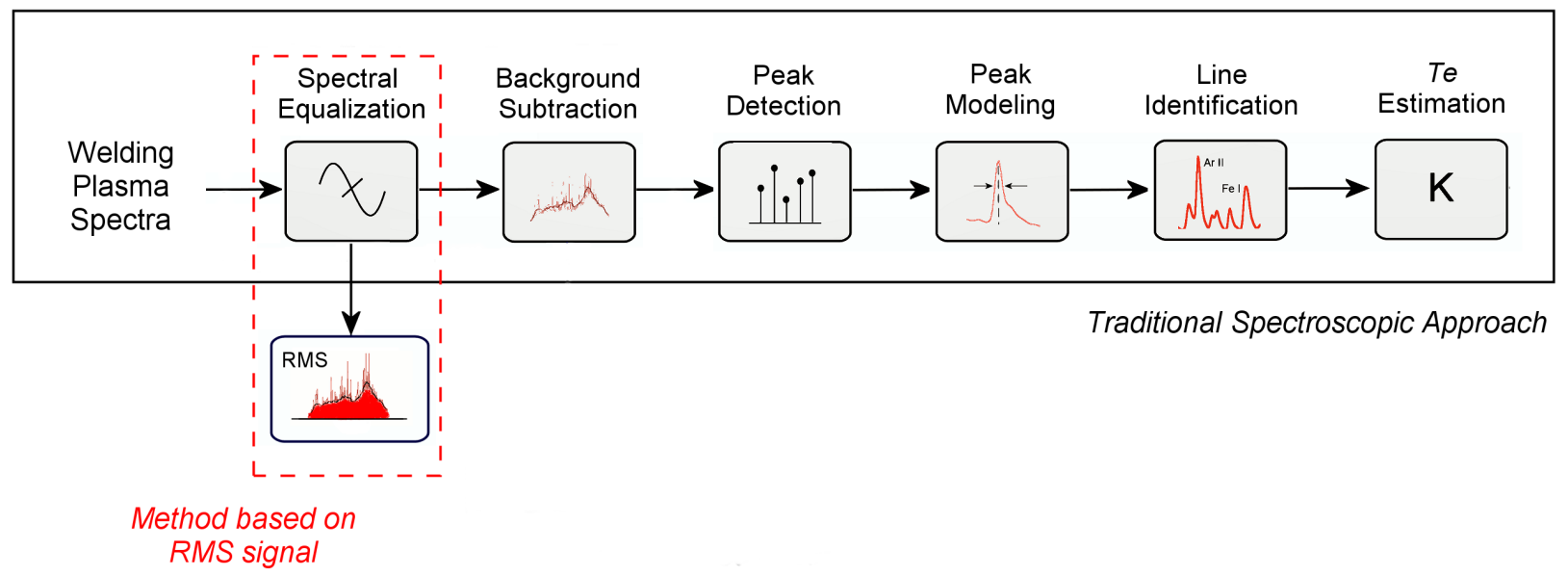

Fig. 1. Processing stages required for spectroscopic welding monitoring.

\section{EXPERIMENTAL ISSUES}

Several experimental tests were performed in the laboratory with a TIG (Tungsten Inert Gas) arc-welding system from Kemppi. The plates (AISI-304 stainless-steel) to be welded were fixed to a positioning system, given that the welding torch was fixed in the setup. The plasma radiation originated during the process was captured with a $50 \mu \mathrm{m}$ core diameter fiber (Ocean Optics P50-UVVIS) that delivered the light to a CCD-spectrometer with spectral range from 195 to $535 \mathrm{~nm}$ (Ocean Optics USB2000). A detail from the experimental setup is shown in Figure 2.

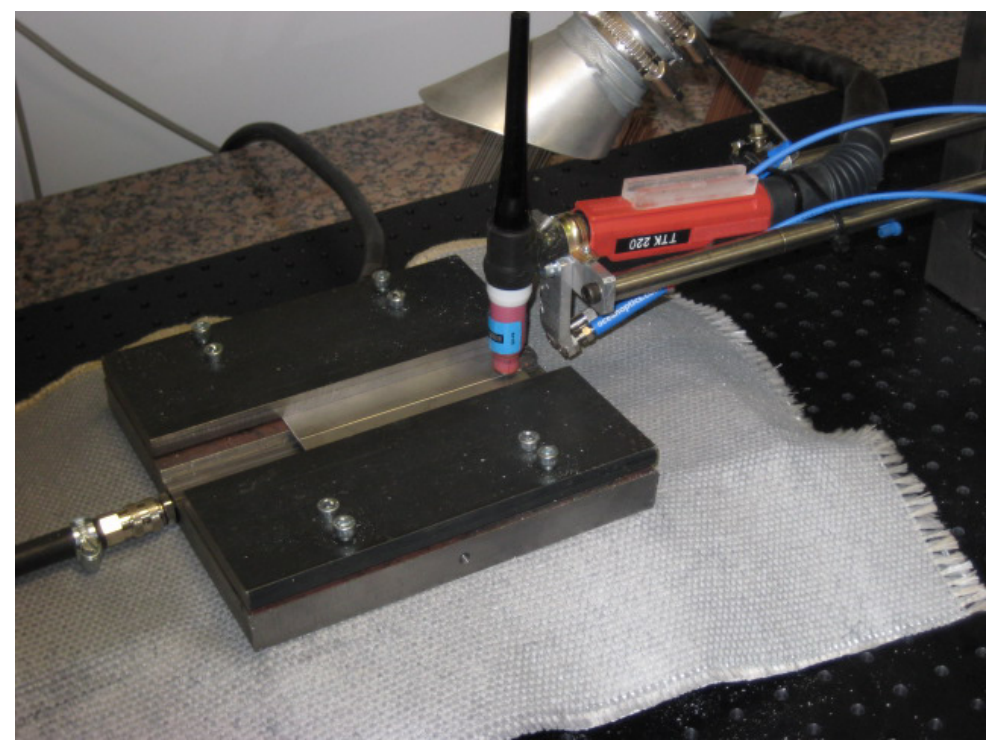

Fig. 2. Detail from the experimental welding setup: welding torch, positioning system with plates and optical fiber. 
Argon was used as shielding gas during the experiments and two different gas sources were used: the welding torch and a secondary output that can be seen on the right side of the image connected to the positioning system. The purpose of this gas intake is to provide a flow of shielding gas to the backside of the plates. Precisely, the defects presented in Figure 3 are related to this back-gas shielding. Figures 3 (a) and (b) show a seam performed with a welding current of 80 A and a speed of $7 \mathrm{~mm} / \mathrm{s}$, where two shortages of the back-gas flow have been performed. The effect of these shortages can be clearly appreciated in Figure 3 (b) (bottom), and two defective sections have been highlighted in Figure 3 (a). The first discontinuity was probably generated by a poor aligment of the plates, while the second was due to the effect of the second back-gas shortage. Figure 3 (c) displays the estimated plasma RMS signal, where a peak appears associated with the second defect. Figure 3 (d) presents the result of using Equation (2), but instead of determining $T_{e}$, the profile directly shows the quotient between the intensities of the chosen Ar II emision lines (480.5 and $488 \mathrm{~nm}$ ). In this case the resulting monitoring parameter does not exhibit any perturbation vinculated to the defect at $x \approx 7 \mathrm{~cm}$.

The defects observed in Figures 3 (e) and (f) were created by an excesive back gas flow, being the welding current pulsed at $5 \mathrm{~Hz}(100$ and $45 \mathrm{~A}$ ), and the welding speed $3 \mathrm{~mm} / \mathrm{s}$. There are clear imperfections on the top of the seam (bead-on-plate), and also on the back, where 5 discontinuities can be appreciated (located at the positions of the back-gas intakes below the plate). Although both the RMS and $I_{1} / I_{2}$ profiles present perturbations associated with the defects, the sensitivity is better in the former.

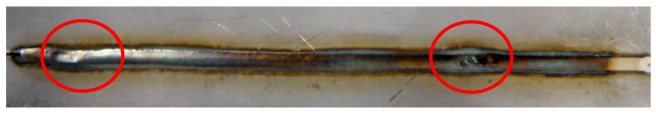

a)

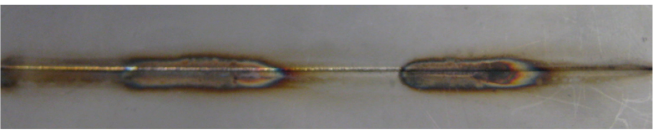

b)
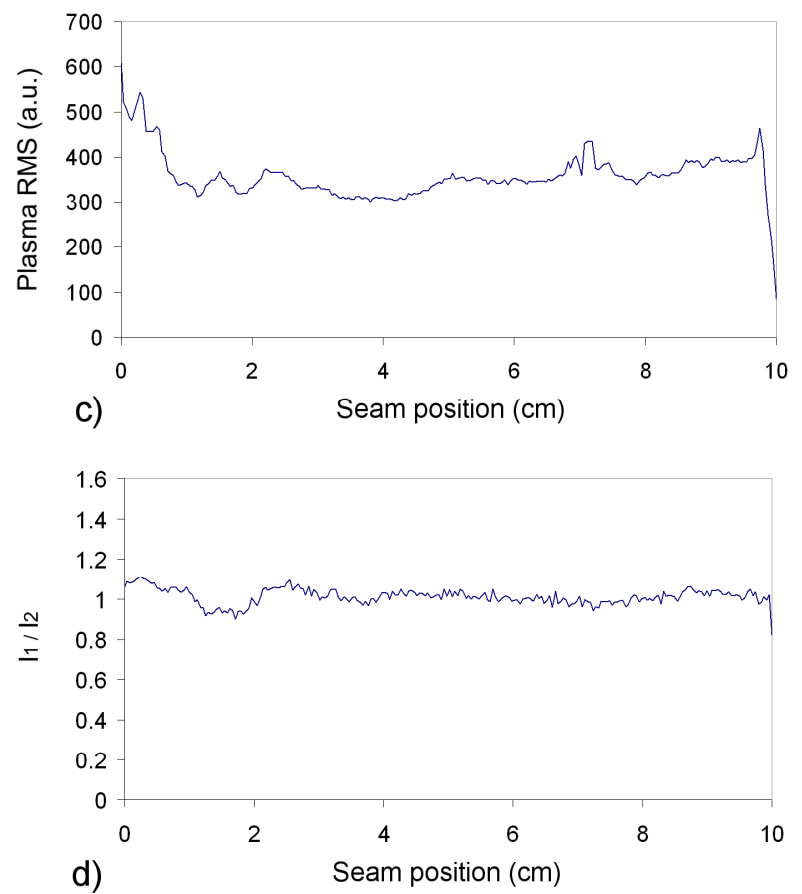

e)

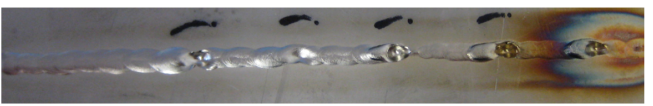

f)
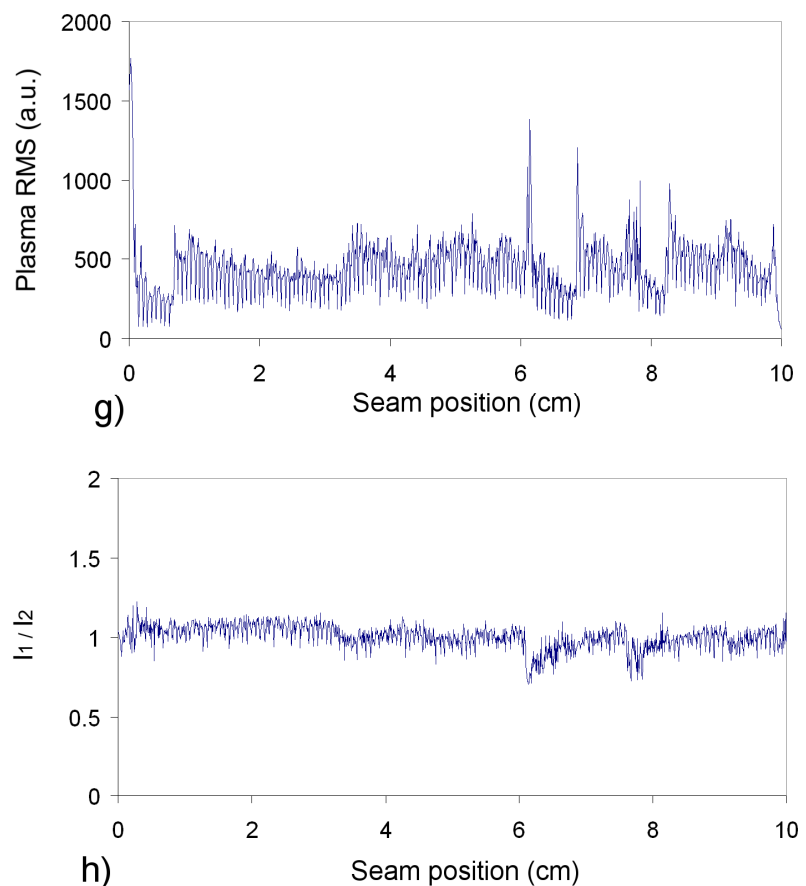

Fig. 3. RMS and $I_{1} / I_{2}$ profiles for defects generated by perturbations on the shielding back-gas flow. 


\begin{tabular}{cc|cc}
\hline \multicolumn{2}{c|}{ Inconel 718 } & \multicolumn{2}{c}{ Titanium 6Al-4V } \\
Element & \% participation & Element & \% participation \\
\hline $\mathrm{Fe}$ & Balanced & $\mathrm{Ti}$ & Balanced \\
$\mathrm{Ni}(+\mathrm{Co})$ & $50-55$ & $\mathrm{Al}$ & $5.5-6.75$ \\
$\mathrm{Cr}$ & $17-21$ & $\mathrm{~V}$ & $3.5-4.5$ \\
$\mathrm{Mo}$ & $2.8-3.3$ & $\mathrm{C}$ & $0.08 \max$ \\
$\mathrm{Nb}(+\mathrm{Ta})$ & $4.75-5.5$ & $\mathrm{H}$ & $0.015 \max$ \\
$\mathrm{Ti}$ & $0.65-1.15$ & $\mathrm{Fe}$ & $0.25 \max$ \\
$\mathrm{Al}$ & $0.2-0.8$ & $\mathrm{~N}$ & $0.05 \max$ \\
$\mathrm{Si}$ & 0.35 & 0 & $0.2 \max$ \\
$\mathrm{Mn}$ & 0.35 & & \\
\hline
\end{tabular}

Table 1.Composition of Inconel 718 and Titanium 6Al-4V.
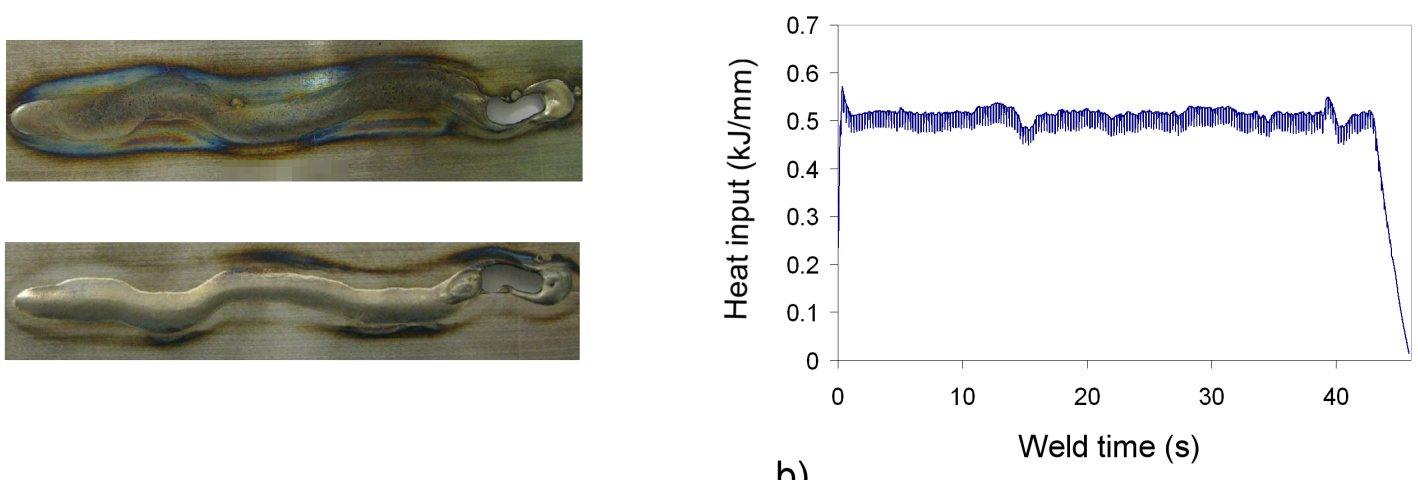

a)

b)
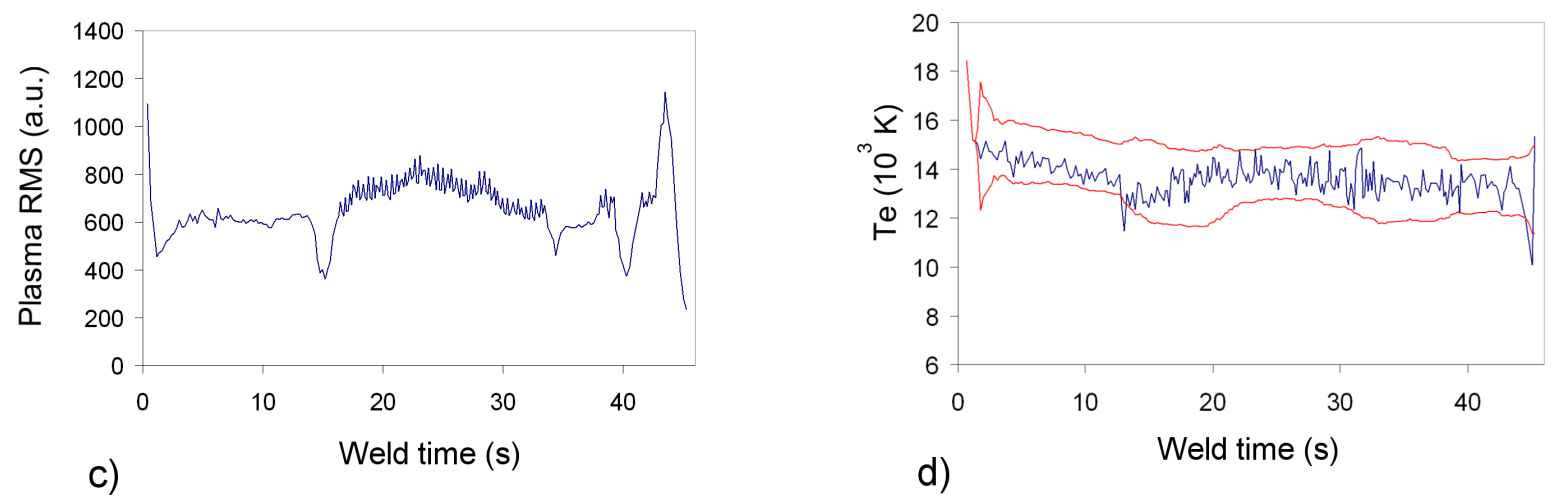

Fig. 4. Defect on Inconel-718 (trajectory deviation) 
A field test result is studied in Figure 4, where a trajectory deviation was intentionally provoked during the arcwelding of two Inconel-718 plates with welding currents of 115 and 45 A (high and low currents), a welding speed of $180 \mathrm{~mm} / \mathrm{min}$, a filler wire speed of $700 \mathrm{~mm} / \mathrm{min}$, a shielding gas flow rate of $10 \mathrm{l} / \mathrm{min}$ and a stick-out (distance between the electrode tip and the plate) of $1.08 \mathrm{~mm}$. The resulting seam is shown in Figure 4 (a) (top and bottom), and the associated heat input profile is depicted in Figure 4 (b). It was determined as the product between the RMS values of both voltage and current of the process, and divided by the torch speed (these data were provided by ROBOTIKER). Some subtle perturbations can be found on the profile, especially at $x \approx 15 \mathrm{~s}$. The plasma RMS signal is depicted in Figure 4 (c), where the indications of a defective seam are more obvious. A $T_{e}$ profile has been also calculated using two Ar II emission lines (at 461 and $488 \mathrm{~nm}$ ), where again the strongest perturbation on the profile is located at $x \approx 15 \mathrm{~s}$. The comparison of these three profiles in terms of sensitivity gives rise to the conclusion that the RMS signal offers a better performance in this regard.

Figure 5 (a) shows the top and the corresponding X-ray image of a seam were an excessive welding current was employed. Several irregularities can be appreciated, as well as the clear peaks that appear on the RMS profile depicted in Figure 5 (b).

A different kind of defect is analyzed in Figure 6, where oil was placed on the position where the seam was performed on a Titanium $6 \mathrm{Al}-4 \mathrm{~V}$ plate. The defective section can be clearly observed in Figure 6 (a) (top of the seam), and the heat input profile also exhibits a distinctive variation on that location. This defect is more obvious on the plasma RMS signal, which starts to decrease from $x \approx 15 \mathrm{~s}$. This effect could be explained by the presence of oil also in that section, what would perturbate the process even if no clear defect is to be seen.
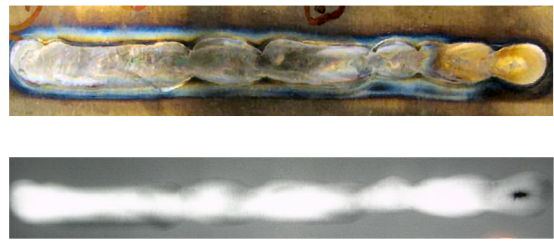

a)

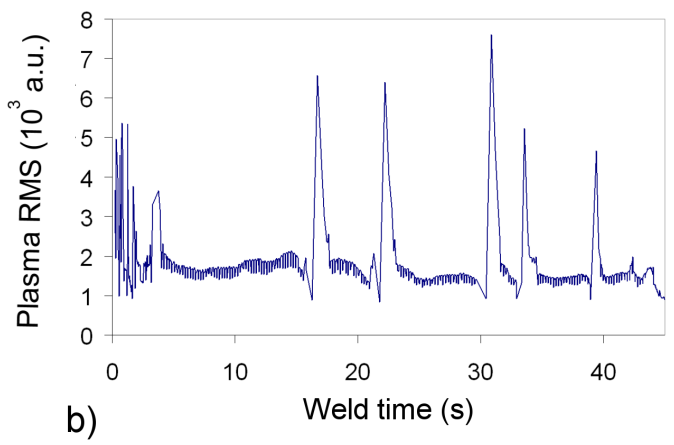

Fig. 5. Defect on Inconel-718 (welding current too high). 


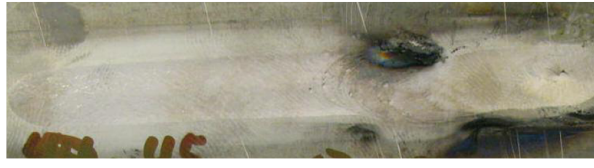

a)

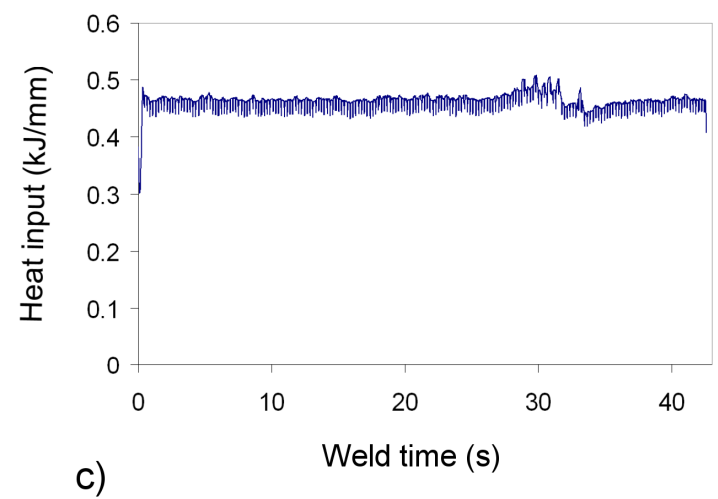

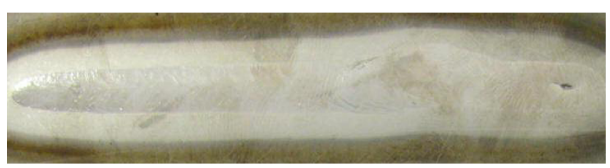

b)

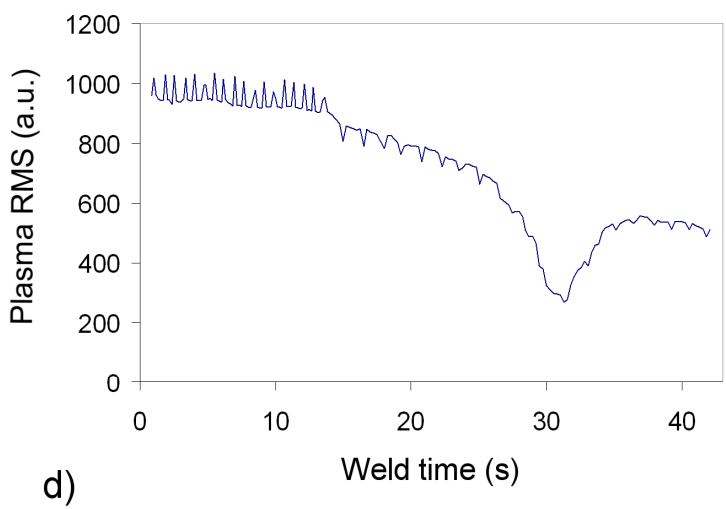

Fig. 6. Defect on Titanium (presence of oil).

\section{CONCLUSIONS}

A new spectroscopic parameter for on-line monitoring of arc-welding processes has been presented in this paper. This new solution is based on the determination of the plasma RMS signal, whose calculation can be performed with a reduced computational cost in comparison to the use of the plasma electronic temperature $T_{e}$. It is directly related to the arc power and, consequently, to the process heat input, although the analyses performed have shown that the latter exhibits a poorer sensitivity in terms of defect detection. The feasibility of the proposed solution has been demonstrated by means of both experimental and field tests on different materials: stainless steel, Inconel and Titanium. In addition various defects have been generated, like perturbations on the shielding back-gas flow, trajectory deviations, inadequate welding currents or lack of cleanliness on the joint. There are still some issues to investigate, like the possibility of using smaller spectral windows than the one provided by the spectrometer. In this regard a feature selection algorithm like SFFS (Sequential Forward Floating Selection) could be chosen to perform an optimal selection of the spectral range in terms of defect detection.

\section{ACKNOWLEDGEMENTS}

This work has been co-supported by the Spanish TEC'2007-67987-C02-01 projects. Authors want to thank J.J. Valdiande for his valuable help during the experimental tests, as well as Jon Magunazelaia (ITP) and Juanma Etayo (ROBOTIKER) for their help and data provided regarding the field tests. 


\section{REFERENCES}

[1] Sibillano, T., Ancona, A., Berardi, V. and Lugara, P.M., "Real-time monitoring of laser welding by correlation analysis: The case of AA5083," Optics and Lasers in Engineering 45(10), 1005-1009 (2007).

[2] Mirapeix, J., Cobo, A., González, D.A., Lopez-Higuera, J.M., "Plasma spectroscopy analysis technique based on optimization algorithms and spectral synthesis," Optics Express 15, 1884-97 (2007).

[3] Mirapeix, J., Cobo, A., Fernandez, S., Cardoso, R., Lopez-Higuera, J.M., "Spectroscopic analysis of the plasma continuum radiation for on-line arc-welding defect detection," J. Phys. D 41, 135202-10 (2006).

[4] Garcia-Allende, P.B., Mirapeix, J., Conde, O.M., Cobo, A. and Lopez-Higuera, J.M., "Defect detection in arcwelding processes by means of the line-to-continuum method and feature selection," Sensors 9(10), 7753-7770 (2009).

[5] Wang, C., Hu, L., Hu, X. and Liu, J., "Relationship between plasma optic signal and penetration depth for partialpenetration laser welding," Chin J Mech Eng Engl Ed 19, 109-12 (2006).

[6] Griem, H.R., [Principles of Plasma Spectroscopy], Cambridge University Press, Cambridge (1997).

[7] National Institute for Standards and Technology (NIST) atomic spectra database (http://physics.nist.gov/cgibin/AtData/main_asd). 